\begin{tabular}{lccc} 
VERSITA & GOSPODARKA & SUROWCAMI & MINERALNYMI \\
\hline \multirow{2}{*}{ Tom 29} & 2013 & Zeszyt 4 \\
& & DOI 10.2478/gospo-2013-0041 &
\end{tabular}

\title{
New potential source of rare earth elements
}

\begin{abstract}
Introduction
Rare earth elements (REE's) are a vital, yet scarce resource which plays a particularly significant role in developed countries and their technologically advanced economies. Most of the world's currently known deposits lie in China, granting this country the status of a monopolist supplier. This drives the prices of REE's up, causing tensions in the international markets. These tensions recently intensified after China curbed the illegal exploitation of REE's, limiting the availability of these resources even further. Recognizing the importance of securing the supply of REE's, both the European Union (EU) and the United States (US) have undertaken steps to diversify the sources of REE's. The EU has defined critical metals as rare-earth elements, as well as the platinum group of metals platinum, palladium, iridium, rhodium, ruthenium, osmium (Critical 2010). The supply of raw materials, especially critical mineral commodities, has been discussed widely by both European (EU Resolution 2011) and Polish (Radwanek-Bąk 2011; Smakowski 2011) scientists and politicians over the last decade. In a recently adopted resolution (2011), the European Union Parliament "Welcomes the proposal for EU diplomacy on RM and REE with the aim of establishing an international regulatory platform, ensuring access to and supply of RM, especially those considered critical, ensuring open global markets and
\end{abstract}

* Prof. dr hab., **** Mgr, Wydział Geologii Uniwersytet Warszawski; Instytut Ceramiki i Materiałów Budowlanych Warszawa; e-mail: krzysztof.szamalek@uw.edu.pl; beata.marciniak@student.uw.edu.pl

** Mgr, Instytut Ceramiki i Materiałów Budowlanych Warszawa; e-mail: g.konopka@icimb.pl

*** Mgr, Wydział Geologii Uniwersytet Warszawski; e-mail: k.zglinicki@student.uw.edu.pl 
promoting international cooperation on sustainable extraction of RM and an efficient use of resources based on mutual interests; underlines, in this context, the need to establish a strong dialogue in the field of RM diplomacy between industrialized, newly industrializing, and resource-rich developing countries, with a view also to promoting human rights, good governance, regional stability and preventing the risk of resource-based conflicts".

Mineral security is now considered one of the key issues of the $21^{\text {st }}$ century (Szamałek 2011a, b; Galos et al. 2012a, b, c). There are new directions in mineral sector activity aimed at securing the supply of resources, such as an increase in exploration programs, use of new mineral resources, introduction of new mineral extraction technologies, and production of new generation mineral commodities. Such new policies are introduced mainly in the US. In 2013, the United States Geological Survey (USGS) started analyzing old and out-of-operation mines, as well as mining and industrial waste, to establish their potential as sources of critical metals (Mining 2013a). Currently, only one US mine supplies REE's (southern California Molycorp Minerals Company). The relationship of supply to demand for heavy rare earth elements (HREE's) is negative in the US and many other industrialized countries. This offers an incentive for identifying new sources of their supply.

Even though the supply of light rare earth elements (LREE) for the US market is less complicated - apart from Molycorp Minerals, they can also be imported from Australia (Lynas Corp, Mt. Weld deposit) - new sources of REE's are necessary. Some companies conduct widespread exploration projects - among them Canadian Avalon Rare Metals, working on the Nechalacho deposit in Canada's Northwest Territories. Should this endeavor succeed, Nechalacho will become Canada's first rare earth mine. This deposit is described as the largest deposit of HREE's outside China (Mining 2013b). The Avalon Company's Prefeasibility Study reported recoveries of $89.7 \% \mathrm{ZrO}_{2}, 79.5 \%$ for all rare earth oxides (REO's), $68.9 \%$ for niobium oxide $\left(\mathrm{Nb}_{2} \mathrm{O}_{5}\right)$, and $63 \%$ for tantalum oxide $\left(\mathrm{Ta}_{2} \mathrm{O}_{5}\right)$.

Until recently, Chinese deposits of REE's (mainly HREE's) were illegally extracted and exported. These unlawful activities were, however, recently curbed by the Chinese government, causing a drop in REE exports and therefore aggravating the tension in the international REE market. Southwest Asia, and Indonesia in particular, have an excellent geopotential of mineral commodities. There are many operating mines containing accessory minerals and elements associated in particular with cassiterite deposits (Szamałek et al. 2013). The Australian Lynas Corp. already operates in Malaysia. The company's ambition is to become the world's largest supplier of REE's (alongside China). Within a decade, oceanic polymetallic massive's containing sulphides (Szamałek et al. 2011) and deep sea muds (Kato et al. 2011) may become new sources of REE's as well as other metals (including critical metals).

This paper discusses tailings obtained in cassiterite extraction on Bangka Island (Indonesia). The first cassiterite extractions on Bangka on an industrial scale were conducted by Chinese companies as early as 1710 . During the $19^{\text {th }}$ and $20^{\text {th }}$ centuries, Indonesia was one of the world's major cassiterite producers. Currently, Indonesia is the second largest tin producer in the world behind China (Schwartz et. al. 1995; minerals.usgs 2013). Indonesian 
cassiterite extraction from offshore and onshore deposits has increased significantly since 2000. Cassiterite is a source of tin. Gravity separation of cassiterite generates large quantities of tailings. The goal of the research conducted in this study was to determine the chemical and mineralogical composition of the tailings and to determine their potential as a source of mineral commodities, especially of REE's.

\section{Materials and Methods}

The research was conducted through cooperation between the Faculty of Geology of Warsaw University and the PT Bumi Bangka Makmur exploring company. Between December 2012 and March 2013, over 450 samples were collected during field surveys on Bangka and Beilitung Islands (Fig. 1). Among the samples are cassiterite sands, the preliminary concentrate of heavy minerals, cassiterite concentrate, and tailing containing heavy minerals lighter than cassiterite $\left(<6.8 \mathrm{~g} / \mathrm{cm}^{3}\right)$. Additionally, slag samples formed from the tin smelting process were collected. The samples were collected from areas (Fig. 1) connected to extraction and processing of cassiterite sands. Sample analyses were conducted in the laboratory of the Institute of Ceramics and Building Materials (Poland), as well as at the Faculty of Geology, Warsaw University.

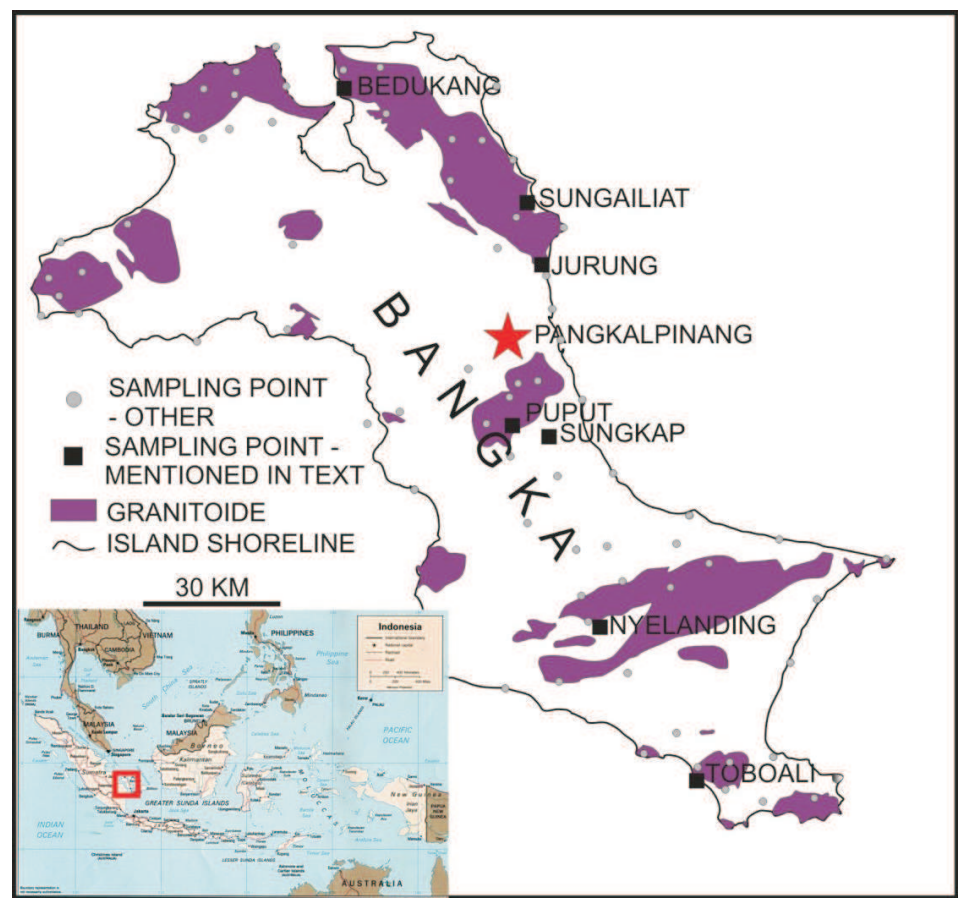

Fig. 1. Location of collected samples (Geological map of Bankga 1994, simplified)

Rys. 1. Lokalizacja miejsc pobrania próbek (Geological map of Bankga 1994, uproszczona) 
This paper presents the preliminary results obtained from analyzing 30 selected samples of bulk cassiterite ores and post-processing tailings. The rock samples were dried and powdered in an agate mortar or in a Herzog disc mill with a tungsten pan. Twenty-nine samples were analyzed on a Bruker AXS D8 Davinci diffractometer equipped with a Lynx Eye PSD detector, automated optics, Cu lamp, and Ni K $\beta$ filter. Settings included 5-1,000 $2 \Theta$ range, primary slit 0.30 , secondary slit 1.50 , soller slits 2.50 , counting time $2 \mathrm{~s} / \mathrm{step}$, and step size $0.0102 \Theta$. Crystalline phases were identified in Bruker Evaluation v.2 software with ICDD PDF-2 v. 2007 and PDF 4+ v. 2011 databases. Quantitative phase analysis was conducted according to the Rietveld method in Bruker Topas v. 4.2 software. Zero shift and instrument factor of the diffractometer were measured on a NIST standard LaB6 660b. Major and minor element identification was conducted on a Panalytical Axios Minerals spectrometer (WD-XRF). Samples were prepared as pressed pellets, following grinding in a Herzog disc mill. Semi-quantitative assay was conducted based on implementation of the IQ+ automated method which relies on fundamental parameters modeling, combined with one-point calibration on a standard sample.

Selected samples were observed under the FEI Nova NANOSEM 200, JEOL JSM-6380LA Scanning Electron Microscope (SEM), and a Nikon binocular for visual light imaging. Mineral grains for SEM were prepared on a carbon film. Uncoated samples were analyzed in low-vacuum mode. Samples coated with $20 \mathrm{~nm}$ of Au were analyzed in high-vacuum $(<10-3 \mathrm{~Pa})$. A BSE (Back Scattered Electron) detector was used. Qualitative chemical analysis with an Energy Dispersive Spectrometer (EDS - model EDAX Apollo X) was applied to differentiate between various minerals in the mixtures. In order to obtain a standardized chemical microanalysis, a different procedure was applied. Samples were included in epoxy resin AralditeTM and polished with a diamond suspension. A $30 \mathrm{~nm}$ layer of carbon was sputtered onto the samples. Prior to measurements, calibration was performed according to standards prepared by MAC (apatite, cyanite, oxides and fluorides of REE, thorium oxide, uranium oxide, lead oxide, yttrium-aluminum garnet). $\mathrm{X}$-ray characteristic lines from the measured samples were registered with the EDAX Apollo-X EDS detector. Spectra were analyzed and deconvoluted in the EDAX Genesis software.

\section{Processing of cassiterite raw material}

Processing cassiterite raw materials is conducted on Bangka Island by two major state owned companies, (i) PT Timah and (ii) PT Koba Tin, which possess the required equipment, and by local miners and compradors (who often use simple, primitive technique and tools). The processing of cassiterite consists of a few stages. The goal of the process is to remove barren rocks and impurities. The preliminary cycles of processing are conducted in situ (in mine) or in processing plants. During these processes, a semi-finished product (concentrate) and waste (tailings) are formed. Cassiterite concentrate processing consists of two basic 
stages. The first includes preliminary ore enrichment in the mine, and the second is enrichment of the cassiterite concentrate at a specialized plant.

In mining excavation, cassiterite ore is washed out using pressurized water. The outwash mixture of water and ore flows down into the excavation pit, from where it is sucked by suction pumps and then channeled into wooden washing cradles. In this process, two types of wooden washing cradles are used - single-stage in very small open pits, and three-stage used in large mine excavations. The wooden washing cradles have thresholds to stop the sediment. During initial water segregation, lighter minerals and clay are eliminated. After this process, heavy minerals fall to the cradle bottom, forming the concentrate which is collected, and tailings which are then moved to a storage area. This enrichment process is ineffective because a significant part of the cassiterite ends up mixed in with the tailings and is moved to waste deposits. These tailing waste dumps are very often mined by illegal miners, who obtain the secondary mineral concentrate and sell it to local compradors. Compradors conduct a multi-treatment enrichment process to achieve a heavy mineral concentrate using concentrating tables. Finally, they obtain cassiterite concentrate and tailings containing monazite, zircon, xenotime, ilmenite, and other minerals. The final cassiterite concentrate is sold to local companies for tin smelting in primitive metallurgical furnaces. Cassiterite concentrate is blended with anthracite and limestone, and then subjected to a reduction process in metallurgical furnaces at $1200^{\circ} \mathrm{C}$. As a result, metallic tin and metallurgical scrap are obtained. This scrap is reprocessed two more times to recover dispersed tin. Solid cassiterite-bearing hydrothermal veins are processed using ball grinders for initial rock disintegration.

Thus, tailings are formed in the many stages of enriching the concentrate. Preliminary tailings obtained in situ in open pits contain $99 \%$ of quartz. Therefore, they are not economically significant as a REE source. Tailings formed during the next stages of enrichment are rich in heavy minerals, mainly those lighter than cassiterite (bulk density $<6.8 \mathrm{~g} / \mathrm{cm}^{3}$ ), among them REE-bearing minerals. Some compradors have tried to separate tailings using concentrating tables, hydrocyclones, and other specialized equipment. Unfortunately, the concentrate they obtain is of unsatisfying purity. Current Indonesian legal regulations prohibit selling bulk raw materials, as well as insufficiently processed minerals.

\section{Tailings' REE potential}

Analyzed tailing samples contain minerals which incorporate light REE (LREE: La-Eu) in their crystal structure (Table 1). Among 23 XRD analyzed tailing samples (Fig. 2), 19 contain monazite $\left[(\mathrm{Ce}, \mathrm{La}, \mathrm{Nd}, \mathrm{Th}) \mathrm{PO}_{4}\right]$. Quantitative Rietveld analysis shows that monazite content varies from $1.38 \%_{\mathrm{W}}$ to $21.23 \%_{\mathrm{W}}\left(3.93 \%_{\mathrm{W}}\right.$ average). Twenty samples contain xenotime $\left[\mathrm{YPO}_{4}\right]$, which incorporates mostly HREE $(\mathrm{Gd}-\mathrm{Lu})$. Xenotime content varies from $0.45 \%$ to $17.55 \%_{\mathrm{W}}\left(2.48 \%_{\mathrm{W}}\right.$ average $)$. 
Mineral composition $\left[\%_{\mathrm{w}}\right]$ of selected representative tailing samples determined by XRD

TABELA 1

Skład mineralny $\left[\%_{\mathrm{w}}\right]$ wybranych próbek odpadów przeróbczych określony metodą XRD

\begin{tabular}{|l|c|c|c|c|c|c||}
\hline \hline Mineral/sample location & Nyelanding & Toboali & Sungkap & Bedukang & Jurung & Puput \\
\hline \hline Monazite & $\mathbf{5 . 2 2}$ & $\mathbf{2 . 4 4}$ & $\mathbf{2 1 . 2 3}$ & $\mathbf{1 . 8 2}$ & $\mathbf{1 . 3 8}$ & $\mathbf{7 . 0 7}$ \\
\hline Xenotime & $\mathbf{1 . 7 8}$ & $\mathbf{3 . 2 7}$ & $\mathbf{1 7 . 5 5}$ & $\mathbf{1 . 0 1}$ & $\mathbf{0 . 5 6}$ & $\mathbf{1 . 1 3}$ \\
\hline Zircon & 7.32 & 18.69 & 16.92 & 10.69 & 1.33 & 62.82 \\
\hline Rutile & 19.97 & 5.09 & 3.77 & 4.73 & 2.80 & 1.55 \\
\hline Anatase & 1.92 & 0.34 & 0.92 & 3.16 & 4.82 & 0.11 \\
\hline Pseudorutile & 41.70 & 28.93 & 7.19 & 38.68 & 37.64 & ND \\
\hline Ilmenite & 2.35 & 9.16 & 16.90 & 4.12 & 3.15 & 8.79 \\
\hline Cassiterite & 1.90 & 3.21 & 8.46 & 1.24 & 0.55 & 18.53 \\
\hline Quartz & 17.80 & 24.65 & ND & 23.53 & 19.25 & ND \\
\hline Pyrite & ND & ND & 4.68 & 2.19 & ND & ND \\
\hline Marcasite & ND & ND & 2.39 & 2.42 & ND & ND \\
\hline Turmaline & ND & 4.23 & ND & 6.42 & 18.69 & ND \\
\hline Topaz & ND & ND & ND & ND & 9.84 & ND \\
\hline
\end{tabular}

ND - below detection limit

ND - poniżej poziomu wykrywalności

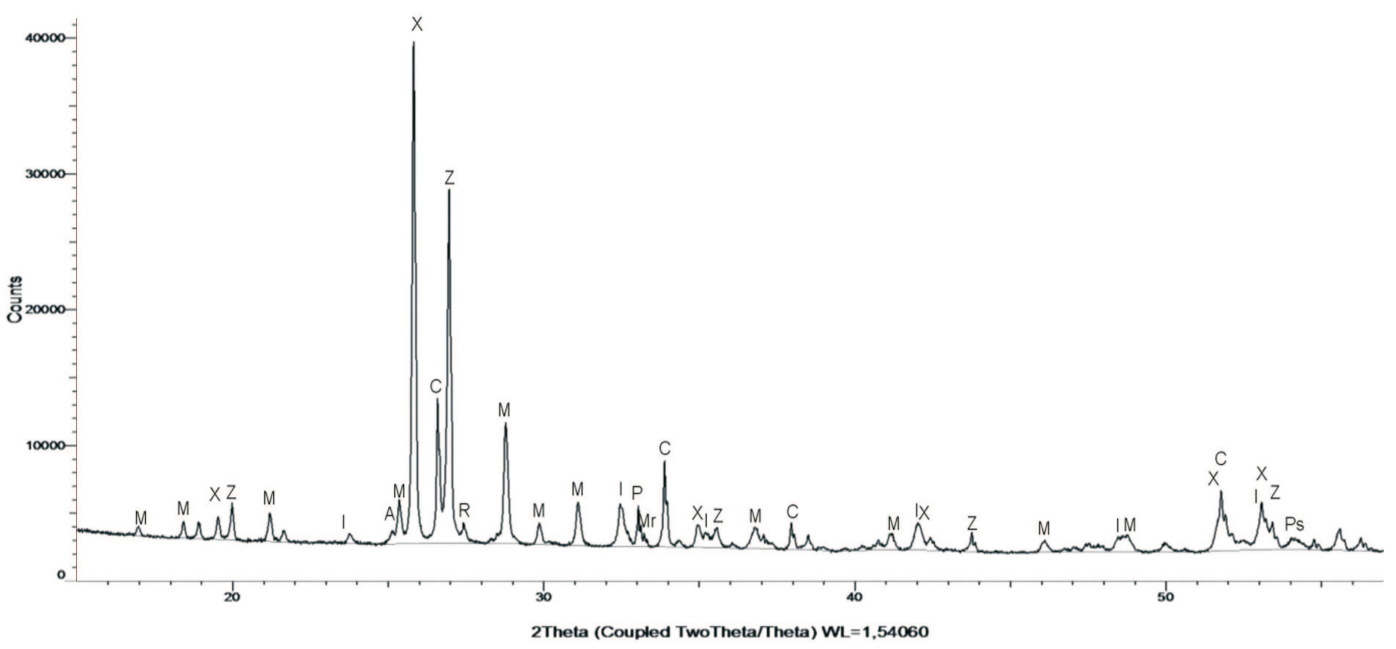

Fig. 2. Mineral composition of tailing from Sungkap, Bangka

$\mathrm{X}$ - xenotime, $\mathrm{M}$ - monazite, $\mathrm{Z}$ - zircon, $\mathrm{I}$ - ilmenite, $\mathrm{A}$ - anatase, $\mathrm{R}$ - rutile, $\mathrm{P}$ - pseurorutile,

$\mathrm{C}$ - cassiterite, $\mathrm{P}$ - piryte, $\mathrm{Mr}$ - marcasite

Rys. 2. Skład mineralny odpadu przeróbczego z Sungkap, Bangka

$\mathrm{X}$ - ksenotym, M - monacyt, Z - cyrkon, I - ilmenite, A - anataz, R - rutyl, P - pseudorutyl, C - kasyteryt,

$\mathrm{P}$ - piryt, $\mathrm{Mr}$ - markasyt 
Lattice parameters of monazite and xenotime from samples containing more than $10 \%_{\mathrm{W}}$ of these minerals were measured according to the Rietveld method based on the crystal structure proposed by Ni et al. (1995) (Table 2 and 3).

TABLE 2

Lattice parameters of monazite determined by XRD analysis

TABELA 2

Parametry komórki elementarnej monacytu oznaczone metodą XRD

\begin{tabular}{|c|c|c||}
\hline Lattice parameters & Monazite from blended concentrate & Monazite from Sungkap (central Bangka) \\
\hline \hline $\mathrm{a}[\AA]$ & $6.80411 \pm 0.00020$ & $6.80571 \pm 0.00061$ \\
\hline $\mathrm{b}[\AA]$ & $7.01671 \pm 0.00020$ & $7.01881 \pm 0.00029$ \\
\hline $\mathrm{c}[\AA]$ & $6.48646 \pm 0.00023$ & $6.48409 \pm 0.00036$ \\
\hline beta $^{0}$ & $103.6825 \pm 0.0029$ & $103.6818 \pm 0.0065$ \\
\hline
\end{tabular}

TABLE 3

Lattice parameters of xenotime determined by XRD analysis

TABELA 3

Parametry komórki elementarnej ksenotymu oznaczone metodą XRD

\begin{tabular}{|c|c|}
\hline Lattice parameters & Sungkap (central Bangka) \\
\hline \hline $\mathrm{a}[\AA]$ & $6.89670 \pm 0.00019$ \\
\hline $\mathrm{b}[\AA]$ & $6.03470 \pm 0.00047$ \\
\hline
\end{tabular}

Selected grain fractions obtained from sieve separation from the sample collected in Toboali (South Bangka) were analyzed by the XRD quantitative Rietveld method (Table 4). Monazite is present in grain fractions less than $1 \mathrm{~mm}$. Xenotime is present in all selected grain fractions, with the exception of the $>2 \mathrm{~mm}$ fraction. The highest concentration of xenotime was measured in the $0.1-0.5 \mathrm{~mm}$ fraction.

Examination of monazite concentrate (Fig. 3) $\left(90.6 \%{ }_{\mathrm{w}}\right.$ monazite and several percent of cassiterite, rutile, xenotime, and pyrite detected by XRD) using a WD-XRF spectrometer in a semi-quantitative mode indicated content of approximately $20.4 \%{ }_{\mathrm{W}} \mathrm{Ce}, 10.8 \%{ }_{\mathrm{W}} \mathrm{La}$, $9.8 \%_{\mathrm{w}} \mathrm{Nd}$, and $2.1 \%_{\mathrm{W}}$ Pr. Additionally, other REE's such as Sm, Gd, Dy, and Y were identified (Table 5). Other elements measured in the concentrate include the actinides thorium (approx. $4.5 \%_{\mathrm{w}}$ ) and uranium (approx. $0.2 \%_{\mathrm{w}}$ ). EDS measurements showed comparable results (Table 6). A low-magnification photo of this concentrate shows pale, yellow to light brown color of monazite grains and the presence of about $15 \%{ }_{\mathrm{W}}$ dark minerals (Fig. 4).

An average chemical composition of 30 monazite grains from the sample of the concentrate was identified in 41 measurement points using standardized EDS microanalysis. Average content of REE's (including yttrium) was calculated to be $59.65 \%$ w. All conducted 


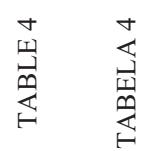

\begin{tabular}{|c|c|c|c|c|c|c|c|c|c|c|c|c|}
\hline & $\begin{array}{l}\Xi \\
\vdots \\
\vdots \\
\vdots \\
\dot{0} \\
\mathrm{D} \\
\mathrm{V}\end{array}$ & 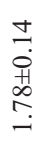 & $\begin{array}{l}n \\
\tilde{\delta} \\
0 \\
+ \\
\text { d } \\
0 \\
0 \\
0\end{array}$ & 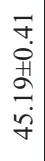 & 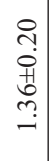 & z & $\mathrm{z}$ & 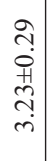 & 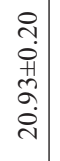 & $\begin{array}{l}\stackrel{+}{~} \\
\stackrel{0}{0} \\
\stackrel{H}{\sim} \\
\stackrel{+}{\sigma} \\
\sigma\end{array}$ & $\begin{array}{l}n \\
n \\
0 \\
+1 \\
\infty \\
\stackrel{1}{0} \\
=\end{array}$ & $\frac{⿱}{\mathfrak{o}}$ \\
\hline & $\begin{array}{c}\vdots \\
\vdots \\
\vdots \\
\dot{0} \\
\frac{1}{5} \\
0 \\
0\end{array}$ & $\begin{array}{l}n \\
\stackrel{n}{0} \\
+1 \\
+ \\
+ \\
\dot{+}\end{array}$ & $\begin{array}{l}\stackrel{n}{0} \\
\stackrel{+}{H} \\
\stackrel{+}{R} \\
-\end{array}$ & 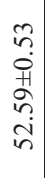 & 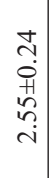 & 之 & 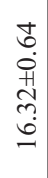 & $\begin{array}{l}\vec{n} \\
0 \\
+1 \\
\stackrel{2}{0} \\
\dot{0}\end{array}$ & $\begin{array}{l}0 \\
0 \\
0 \\
+1 \\
0 \\
\infty \\
\\
i\end{array}$ & 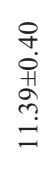 & 之 & $\underset{\sim}{\tilde{\sigma}}$ \\
\hline .0 & 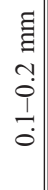 & 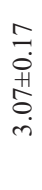 & $\begin{array}{l}m \\
\stackrel{0}{0} \\
\stackrel{H}{0} \\
0 \\
\dot{+}\end{array}$ & 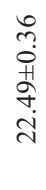 & $\begin{array}{l}\infty \\
\stackrel{0}{0} \\
\stackrel{+}{0} \\
\infty \\
\dot{n}\end{array}$ & 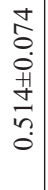 & $\begin{array}{l}\stackrel{2}{2} \\
0 \\
+ \\
\stackrel{+}{2} \\
\dot{m} \\
m\end{array}$ & $\begin{array}{l}\vec{m} \\
0 \\
+ \\
\hat{n} \\
\stackrel{n}{i} \\
\end{array}$ & $\begin{array}{c}0 \\
0 \\
0 \\
0 \\
0 \\
0 \\
i n \\
i\end{array}$ & 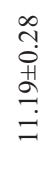 & 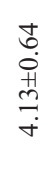 & $\frac{\hat{\sigma}}{\dot{m}}$ \\
\hline.$=$ & $\begin{array}{c}\Xi \\
\vdots \\
n \\
0 \\
\vdots \\
\sim \\
\vdots \\
0\end{array}$ & $\begin{array}{l}\infty \\
\stackrel{\infty}{0} \\
+ \\
\sim \\
\sim \\
\sim\end{array}$ & 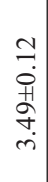 & 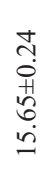 & $\begin{array}{l}\cong \\
0 \\
0 \\
+1 \\
0 \\
0 \\
\dot{r}\end{array}$ & 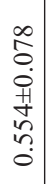 & $\begin{array}{c}n \\
\infty \\
0 \\
+ \\
+ \\
+ \\
\infty \\
\infty \\
N\end{array}$ & $\begin{array}{l}\stackrel{2}{1} \\
\stackrel{0}{0} \\
\stackrel{+}{2} \\
\stackrel{\infty}{\infty}\end{array}$ & $\begin{array}{l}\infty \\
0 \\
0 \\
0 \\
+1 \\
\infty \\
\infty \\
\infty \\
i\end{array}$ & 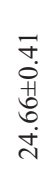 & $\begin{array}{l}\overline{0} \\
0 \\
+ \\
\stackrel{0}{r}\end{array}$ & $\begin{array}{l}q \\
8 \\
8\end{array}$ \\
\hline
\end{tabular}

(3)

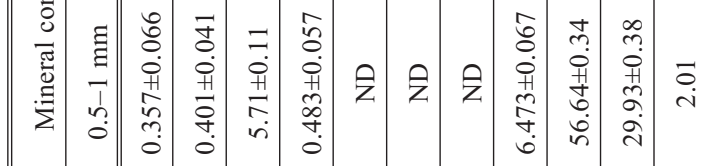

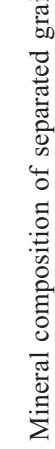

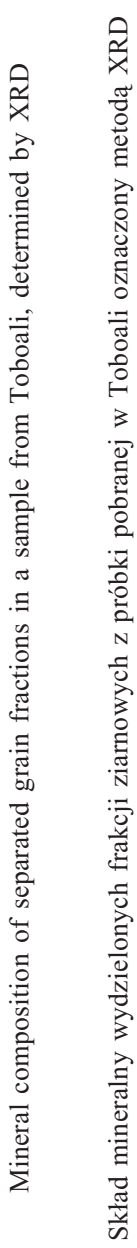

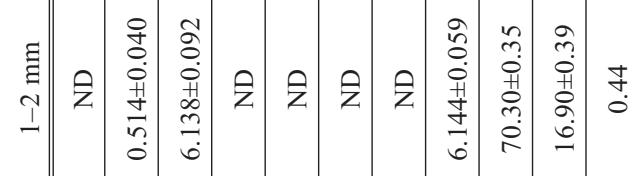

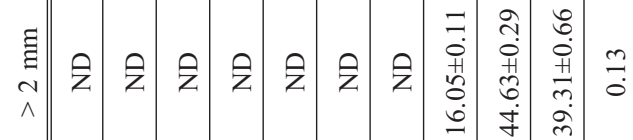

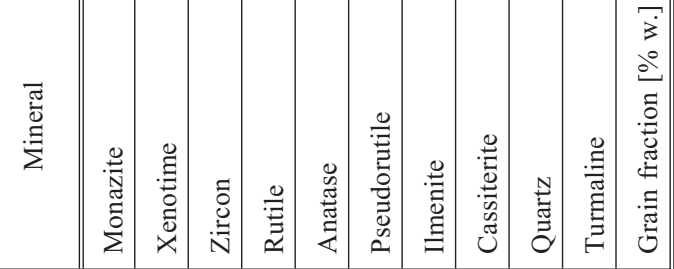

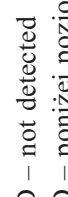

合 


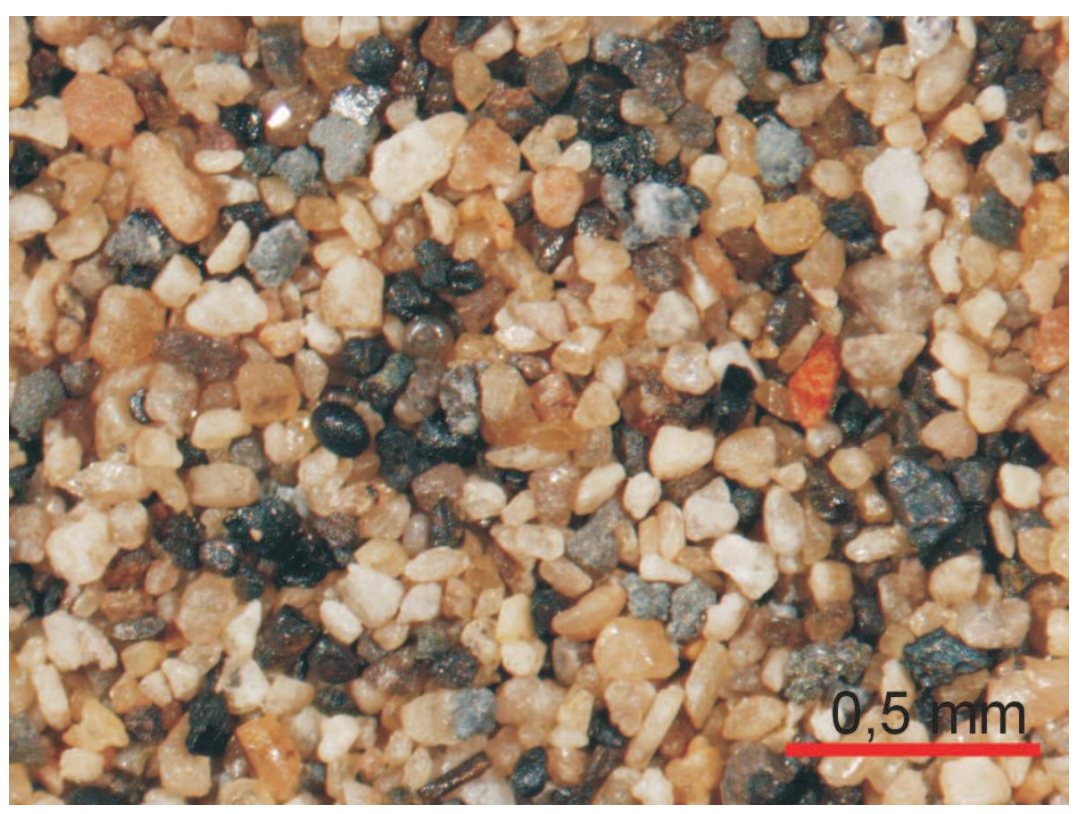

Fig. 3. Monazite concentrate observed under binocular. Phot. K. Zgliniecki

Rys. 3. Koncentrat monacytowy - zdjęcie z binokularu. Fot. K. Zgliniecki

Results of WD-XRF semi-quantitative analysis of monazite concentrate, Bangka

Wyniki półilościowej analizy WD-XRF koncentratu monacytowego, Bangka

\begin{tabular}{||l|c||l|l||l|c||}
\hline \hline \multicolumn{1}{|c|}{ Compound } & \%w. & \multicolumn{1}{|c||}{ Compound } & \%w. & Compound & \% w. \\
\hline \hline $\mathrm{F}$ & 1.34 & $\mathrm{MnO}_{2}$ & 0.12 & $\mathrm{Nd}_{2} \mathrm{O}_{3}$ & 9.84 \\
\hline $\mathrm{MgO}$ & 0.21 & $\mathrm{Fe}_{2} \mathrm{O}_{3}$ & 5.04 & $\mathrm{Sm}_{2} \mathrm{O}_{3}$ & 1.28 \\
\hline $\mathrm{Al}_{2} \mathrm{O}_{3}$ & 0.71 & $\mathrm{Y}_{2} \mathrm{O}_{3}$ & 1.24 & $\mathrm{Gd}_{2} \mathrm{O}_{3}$ & 0.70 \\
\hline $\mathrm{SiO}_{2}$ & 2.41 & $\mathrm{ZrO}_{2}$ & 0.98 & $\mathrm{Dy}_{2} \mathrm{O}_{3}$ & 0.39 \\
\hline $\mathrm{P}_{2} \mathrm{O}_{5}$ & 16.3 & $\mathrm{Nb}_{2} \mathrm{O}_{5}$ & 0.10 & $\mathrm{WO}_{2}{ }^{*}$ & 0.10 \\
\hline $\mathrm{SO}_{3}$ & 4.5 & $\mathrm{SnO}_{2}$ & 8.23 & $\mathrm{ThO}_{2}$ & 4.49 \\
\hline $\mathrm{K}_{2} \mathrm{O}$ & 0.03 & $\mathrm{La}_{2} \mathrm{O}_{3}$ & 10.80 & $\mathrm{U}_{3} \mathrm{O}_{8}$ & 0.18 \\
\hline $\mathrm{CaO}$ & 0.24 & $\mathrm{Ce}_{2} \mathrm{O}_{3}$ & 20.40 & $\mathrm{Sum}$ & 95.5 \\
\hline $\mathrm{TiO}$ & 3.96 & $\mathrm{Pr}_{2} \mathrm{O}_{3}$ & 2.05 & & \\
\hline
\end{tabular}

* Milled in tungsten mortar 
Average oxides concentration measured on selected grains from monazite concentrate, Bangka

TABELA 6

Koncentracja tlenków oznaczona na wybranych ziarnach monacytu, Bangka

\begin{tabular}{|l|c|c||l|c|c|}
\hline \hline OXIDE & AVG $\left[\%_{\mathrm{w}}\right]$ & STD & OXIDE & AVG $\left[\%_{\mathrm{w}}\right]$ & STD \\
\hline \hline $\mathrm{Al}_{2} \mathrm{O}_{3}$ & 0.14 & 0.05 & $\mathrm{La}_{2} \mathrm{O}_{3}$ & 11.98 & 1.18 \\
\hline $\mathrm{SiO}_{2}$ & 1.52 & 0.57 & $\mathrm{Ce}_{2} \mathrm{O}_{3}$ & 29.57 & 1.92 \\
\hline $\mathrm{Y}_{2} \mathrm{O}_{3}$ & 2.60 & 0.69 & $\mathrm{Pr}_{2} \mathrm{O}_{3}$ & 2.99 & 0.20 \\
\hline $\mathrm{P}_{2} \mathrm{O}_{5}$ & 29.79 & 0.88 & $\mathrm{Nd}_{2} \mathrm{O}_{3}$ & 10.23 & 0.97 \\
\hline $\mathrm{ThO}_{2}$ & 6.70 & 1.99 & $\mathrm{Sm}_{2} \mathrm{O}_{3}$ & 1.31 & 0.49 \\
\hline $\mathrm{UO}_{2}$ & 1.41 & 0.46 & $\mathrm{Gd}_{2} \mathrm{O}_{3}$ & 0.97 & 0.30 \\
\hline $\mathrm{CaO}$ & 0.27 & 0.17 & Sum: & $\mathbf{9 9 . 4 7}$ & $\mathbf{0 . 5 5}$ \\
\hline
\end{tabular}

STD - standard deviation

STD - odchylenie standardowe

AVG - average content

AVG - średnia zawartość

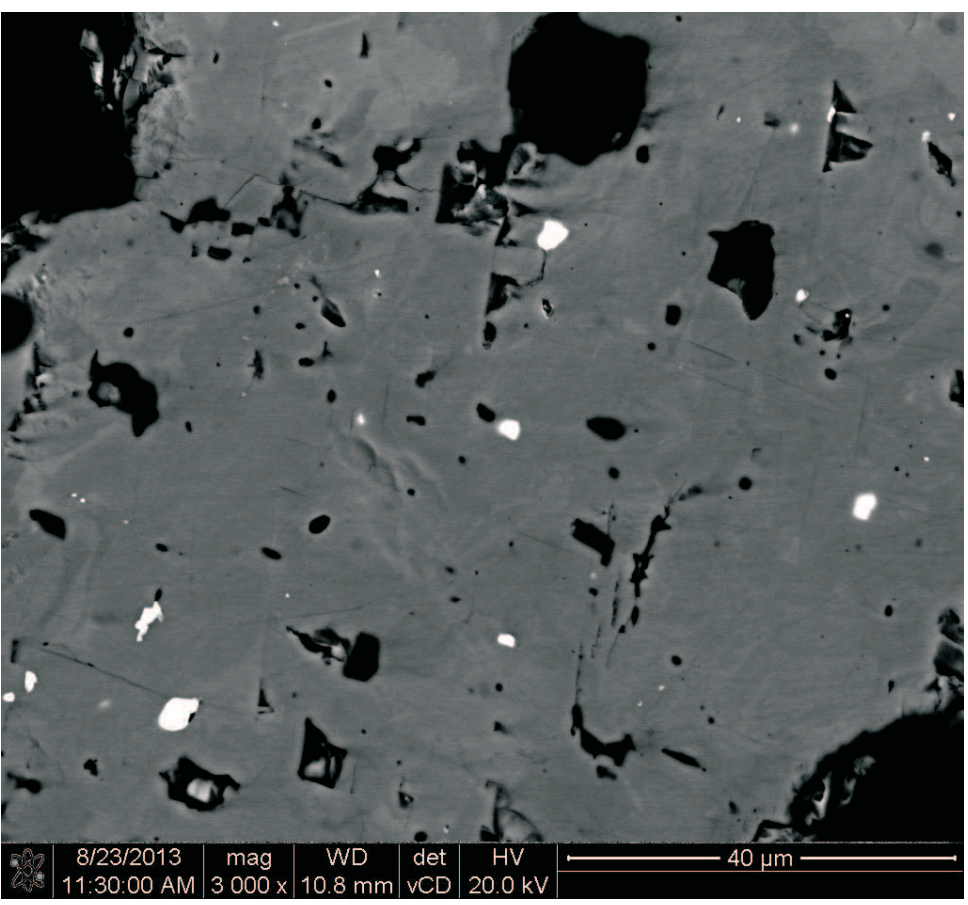

Fig. 4 Monazite grain from Sungailiat. SEM with a BSE detector. Polished sample Rys. 4. Ziarno monacytu z Sungailiat. SEM z BSE detektorem. Próbka polerowana 
chemical microanalyses (also on grains from other locations - unpublished data) have shown that among this group of elements, cerium is always predominant over others (Table 6). Therefore, monazite- $(\mathrm{Ce})$ is the proper name for this mineral. The identified REE concentration declines from cerium, lanthanum, neodymium, to praseodymium, with trace amounts of samarium and gadolinium. Thorium content is always a few times greater than that of uranium.

BSE imaging (Fig. 4) shows that monazite grains have a zoned structure. Lighter parts of monazite images are characterized by a higher concentration of actinides in contrast to the darker parts. Numerous inclusions of thorium silicate and uranium silicate - probably huttonite, being isostructural to monazite - were observed. However, their concentration

Average oxides concentration measured on selected xenotime grains from tailing samples from Toboali and Sungailiat

TABELA 7

Koncentracja tlenków oznaczona na wybranych ziarnach ksenotymu z odpadu przeróbczego z Toboali i Sungailiat

\begin{tabular}{|l|c|c|c|c|}
\hline \multirow{2}{*}{ Oxide } & \multicolumn{2}{|c|}{ Toboali } & \multicolumn{2}{c|}{ Sungailiat } \\
\cline { 2 - 5 } & AVG [\% $\left.{ }_{\mathrm{w}}\right]$ & $\mathrm{STD}$ & AVG $\left.\left[{ }_{\mathrm{w}}\right]\right]$ & STD \\
\hline \hline $\mathrm{SiO}_{2}$ & 0.02 & 0.08 & 0.01 & 0.07 \\
\hline $\mathrm{Y}_{2} \mathrm{O}_{3}$ & 38.62 & 1.20 & 40.55 & 1.73 \\
\hline $\mathrm{P}_{2} \mathrm{O}_{5}$ & 36.71 & 0.70 & 37.75 & 0.80 \\
\hline $\mathrm{PbO}_{2}$ & 0.29 & 0.03 & 0.30 & 0.10 \\
\hline $\mathrm{ThO}_{2}$ & 0.95 & 0.54 & 0.52 & 0.57 \\
\hline $\mathrm{UO}_{2}$ & 0.90 & 0.53 & 0.90 & 0.79 \\
\hline $\mathrm{Nd}_{2} \mathrm{O}_{3}$ & 0.36 & 0.19 & 0.30 & 0.18 \\
\hline $\mathrm{Sm}_{2} \mathrm{O}_{3}$ & 0.86 & 0.41 & 0.64 & 0.13 \\
\hline $\mathrm{Gd}_{2} \mathrm{O}_{3}$ & 3.22 & 1.05 & 2.23 & 0.38 \\
\hline $\mathrm{Tb}_{2} \mathrm{O}_{3}$ & 0.88 & 0.18 & 0.68 & 0.12 \\
\hline $\mathrm{Dy}_{2} \mathrm{O}_{3}$ & 6.13 & 0.73 & 5.48 & 0.64 \\
\hline $\mathrm{Ho}_{2} \mathrm{O}_{3}$ & 1.39 & 0.17 & 1.34 & 0.13 \\
\hline $\mathrm{Er}_{2} \mathrm{O}_{3}$ & 4.25 & 0.69 & 4.34 & 0.42 \\
\hline $\mathrm{Yb}_{2} \mathrm{O}_{3}$ & 4.36 & 0.88 & 4.42 & 0.86 \\
\hline $\mathrm{Lu}_{2} \mathrm{O}_{3}$ & 0.67 & 0.13 & 0.56 & 0.28 \\
\hline $\mathrm{Sum}_{\mathbf{y y y y y}}$ & $\mathbf{9 9 . 6 2}$ & $\mathbf{0 . 8 5}$ & $\mathbf{1 0 0 . 0 2}$ & $\mathbf{1 . 1 5}$ \\
\hline
\end{tabular}

STD - standard deviation

STD - odchylenie standardowe

AVG - average content

AVG - średnia zawartość 
varies greatly between individual grains. BSE imaging reveals that xenotime grains (Fig. 5) are characterized by an irregular domain or, more rarely, concentric zonation. Lighter parts of BSE images also show a higher concentration of actinides ( $U$ and $T h$ ) in the chemical microanalysis. Inclusions of uranium silicate and thorium silicate - probably uranothorite were identified within the monazite grains, more often in the lighter parts than in the darker parts. Monazite and xenotime grains were more porous than the other heavy mineral grains observed on polished samples, as well as on grain surfaces (Fig. 6 and 7).

Chemical microanalysis of selected xenotime grains identified the presence of significant HREE concentration. The content of lanthanide oxides is on average $22.13 \%_{\mathrm{W}}$ of Toboali xenotime and $19.99 \%_{\mathrm{W}}$ of Sungailiat xenotime. Yttrium oxide is present at $38.62 \% \mathrm{w}_{\mathrm{w}}$ and $40.55 \%_{\mathrm{w}}$, respectively. Other critical metals - niobium and tantalum - were identified in slag formed during tin smelting. Uncalibrated EDS analysis determined (besides $\mathrm{Zr}$, Ti, and REE) the presence of $\mathrm{Nb}$ and $\mathrm{Ta}$ in amounts of about $1 \%$ each. Calibrated EDS analysis of cassiterite grains (furnace feed) identified the presence of up to $1 \% \mathrm{TaO}$ and up to $0.25 \%$ $\mathrm{Nb}_{2} \mathrm{O}_{5}$. Mineral phases of niobium and tantalum (i.e. columbite-tantalite) were not identified. Ilmenite grains containing needle-shaped or rectangular phases with elevated $\mathrm{Nb}$ and $\mathrm{Y}$ concentration were identified; however, they were too small to analyze with EDX. $\mathrm{Nb}$ is also present as a substitution in the crystal structure of ilmenite and rutile. $\mathrm{Nb}$ content in rutile is rather constant at about $0.5 \%$, while in ilmenite it is highly variable from below detection limit to $0.3 \%$.

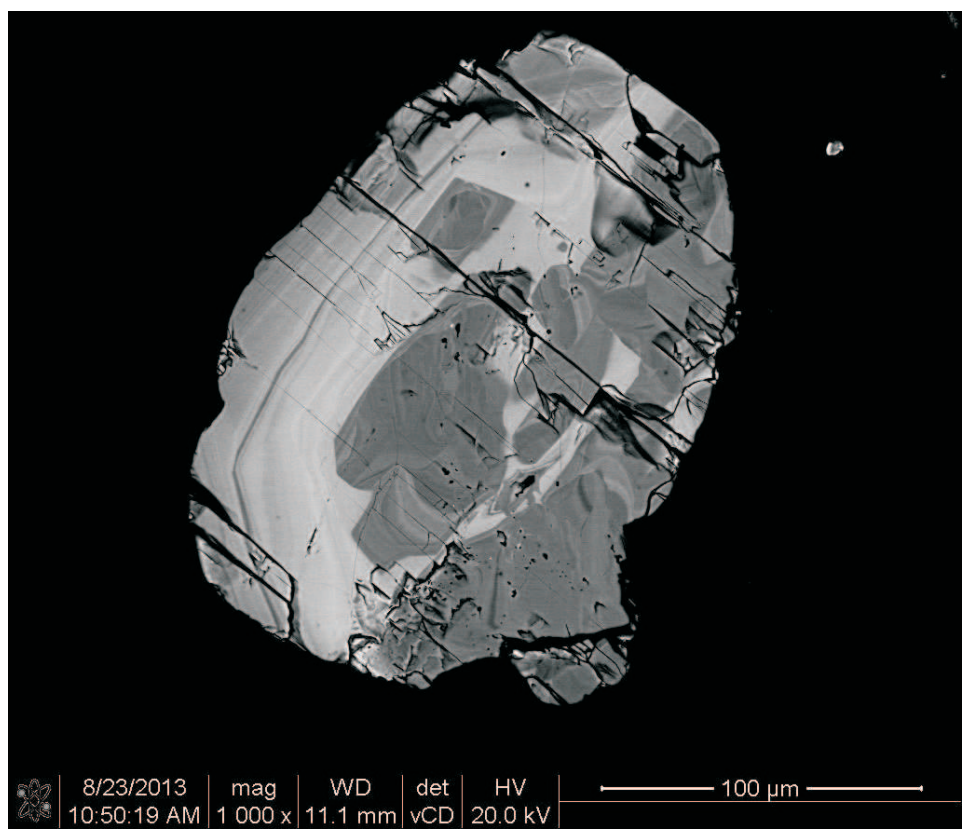

Fig. 5. Xenotime grain from Toboali. SEM with a BSE detector. Polished sample Rys. 5. Ziarno ksenotymu z Toboali. SEM z BSE detektorem. Próbka polerowana 


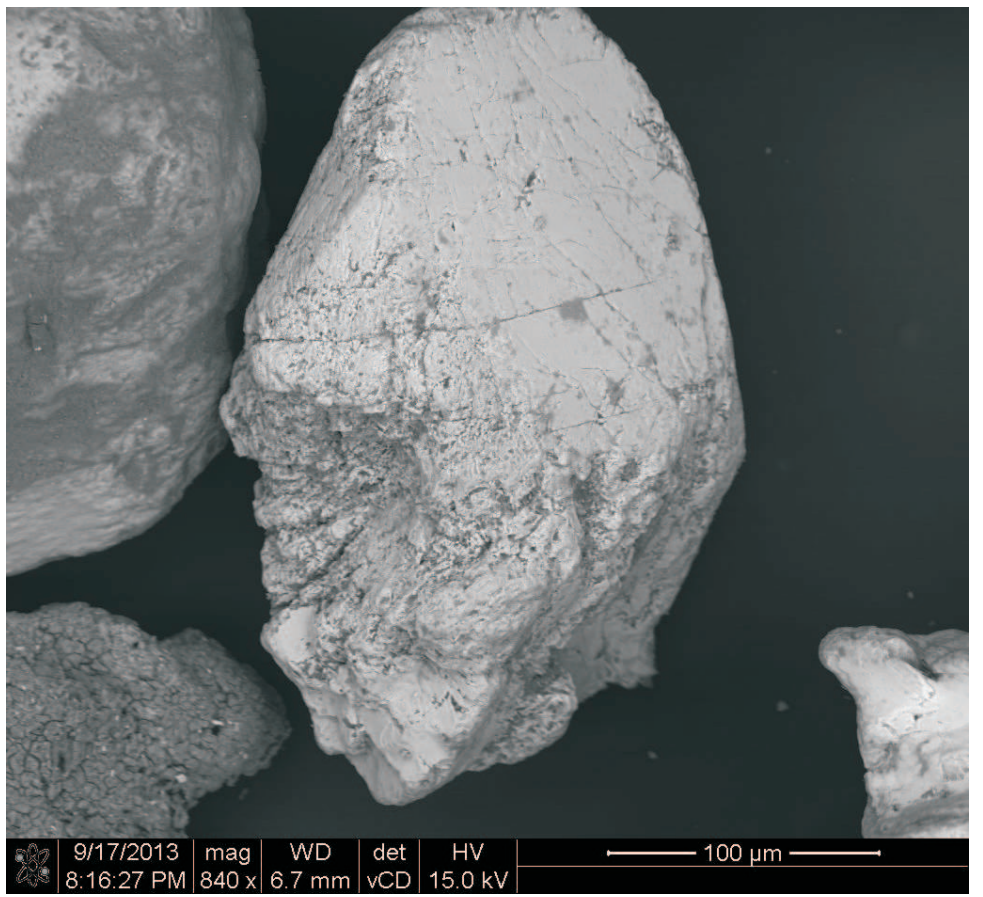

Fig. 6. Typical form of monazite grain from monazite concentrate, Bangka. SEM with a BSE detector Rys. 6. Typowa forma monacytu z koncentratu monacytowego, Bangka. SEM z detektorem BSE

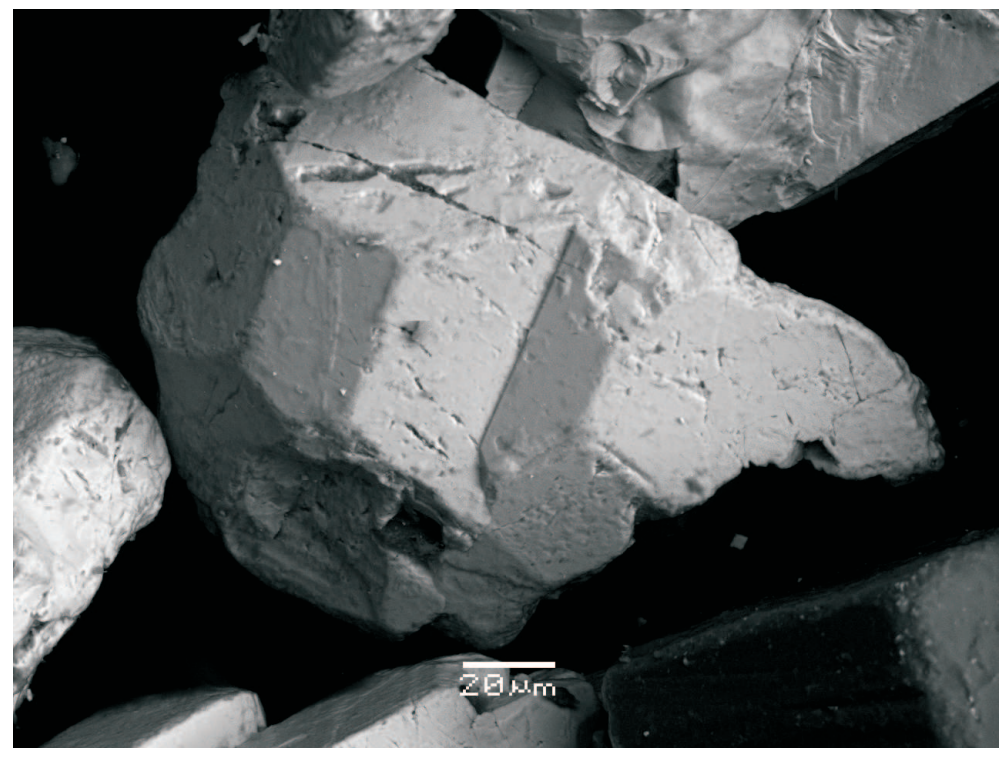

Fig. 7 Automorphic monazite grain from the concentrate. SEM with a BSE detector Rys. 7. Automorficzne ziarno monacytu z koncentratu. SEM z detektorem BSE 


\section{Mineral resource estimation}

Mineral resource estimation is not easy due to the dispersed and partially illegal character of mining and processing of cassiterite ore, as well as the fact that the size and mass of tailings accumulated on various stockpiles in Bangka have not yet been determined. Reliable data indicate that it is possible to obtain about 28,000 tons of high quality (low in quartz) tailings. These values take into account only tailings formed in major tin smelters during the last stage of cassiterite ore processing.

Probably more than a thousand compradors operate on the island, each of them producing about 100 tons of tailings monthly. This means that annually even as much as 1.2 million tons of tailings can be separated. That figure is probably the upper limit of the calculations (Table 8). However, tailings produced by some compradors contain a significant amount of quartz and are thus of lower grade than tailings from tin processing plants. State owned companies, such as PT Timah and PT Koba Tin, have significant amounts of stockpiled tailings which have previously been sold to Malaysia. Knowing the average concentration of monazite and xenotime in the examined samples, as well as the quantity of the tailings, we can calculate the maximum recoverable amount of these minerals (based on the assumption that the separation process is conducted without any losses). The lower limit of annual tailing production is 28,080 tons, as declared by major tin smelters (unpublished data). A realistic estimation of tailing production, based on a thorough field survey, is 180,000 tons annually

TABLE 8

Estimation of annual potential supply of monazite and xenotime depending on various estimations of annual tailing production

TABELA 8

Oszacowanie rocznej potencjalnej podaży monacytu i ksenotymu z wariantowej rocznej produkcji odpadów

\begin{tabular}{|c|c|c|c||}
\hline Mineral [t] & $\begin{array}{c}\text { Annual total tailings } \\
\text { production of 28 080 tons }\end{array}$ & $\begin{array}{c}\text { Annual total tailings } \\
\text { production of 180 000 tons }\end{array}$ & $\begin{array}{c}\text { Annual total tailings } \\
\text { production of 1 200 000 tons }\end{array}$ \\
\hline \hline Monazite & 1106 & 7092 & 47280 \\
\hline Xenotime & 696 & 4464 & 29760 \\
\hline
\end{tabular}

Taking into account said assumption, one can realistically assume that up to 10,000 tons of monazite and xenotime per annum can be recovered from Bangka Island alone. Research on the mineral potential of REE's on the nearby Belitung (Billiton) Island is currently in progress. The USGS reports that annual global monazite concentrate production is above 6,000 tons (besides China and Indonesia); therefore, tailings from Indonesian islands may play a very important role in the international mineral commodities market. 


\section{Discussion}

Primary tin and tungsten mineralization in Indonesia is found in post magmatic rocks such as greisens and veins (Schwartz et al. 1995). Heavy minerals characterized by high mechanical and chemical resistance (including monazite and xenotime) co-exist in placer cassiterite deposits in different parts of the world (Dill et al. 2006; Schwartz et al. 1995). Monazite and xenotime are very common accessory minerals of granites (Maruejol et al. 1990; Gawęda et al. 2009; Förster 1998). The high content of thorium in examined samples is characteristic for monazite of magmatic origin (Zhu, O'Nions 1999). The concentration of HREE's, $U$ and Th, as well as numerous uranothorite inclusions in examined xenotime, indicates a magmatic origin of xenotime (Kositcin et al. 2003). The xenotime HREE to Y ratio is typical for granitoids of S or I type (Förster 1998), which falls into the existing classification of adjacent magmatic plutons (Schwartz et al. 1995).

Our research suggests that REE minerals co-existing with placer cassiterite originated from weathering of granitoids. This complies with other research of SE Asian heavy mineral assemblages, where xenotime and monazite are reported to have originated from Main Range Province granitoids in the Malay Peninsula (Sevastjanova et al. 2012). Main Range-type granitoids are widely distributed on the Indonesian Tin Islands (Schwartz et al. 1995). The chemical composition of monazite and xenotime is similar to the chemical composition of these REE phosphates from Australian placer deposits (Van Emden et al. 1997). In addition, measured lattice parameters comply well with the published data for minerals with highly similar chemical composition (Ni et al. 1995; Mogilevsky et al. 2006). However, the dataset used for these structural calculations was limited. The morphology and structure of REE phosphates is variable. Among them, automorphic, subautomorphic, and xenomorphic grains can be observed, indicating that the distance from the alimentation area was variable. The dominating tabular shape of automorphic monazite crystals indicates a granitic, as opposed to a pegmatitic, provenance (Dill et al. 2012). High secondary porosity of polished grains of monazite and xenotime and their sharp-edged cavities on crystal faces indicate that these minerals have low chemical and mechanical resistance against transportation and weathering in comparison with, e.g., zircon. Monazite is reported to have moderate chemical and very low mechanical resistivity among heavy minerals (Morton, Hallsworth 1999). Poor roundness is typical for phosphates in tropical placers (Dill 2007).

The examined samples of tailings and slag contain significant concentrations of mineral carriers of both LREE's (monazite) and HREE's (xenotime). It is necessary to conduct a technological examination in order to test the potential of obtaining pure monazite and xenotime concentrates. At the same time, it is critical to know the chances for recovering tantalum and niobium, which are present as substitutions in the crystal structure of cassiterite and Ti-minerals. A significant source of these rare elements could be slag. 


\section{Summary and conclusions}

1. Recent political tension and disturbances in the international REE mineral commodities market caused by the Chinese supply monopoly force industrialized countries to launch intensive programs to discover new sources of these elements. Current world REE demand is less than their supply, causing the price of these elements to climb higher. Additionally, unacceptable political and economic barriers to REE trade have been implemented.

2. Securing new sources of REE's is a lengthy process. Exploratory surveys take a lot of time, and a lengthy timeframe also exists before a discovered REE deposit can be mined. A high tech economy demands REE's for further development. Therefore, relevant new mineral resource policy is based on prospecting works concentrated on old, exhausted deposits, closed mines, waste dumps, and slag dumps.

3. Indonesia is a promising source of REE's, as a country with a long history in mining cassiterite. Waste produced during mining, as well as post-processing slag, are potential sources for recovering critical metals. The conducted research determined that tailings from Bangka Island contain significant amounts of monazite, xenotime, zircon, rutile, anatase, pseudorutile, and ilmenite, together with residual cassiterite. Apart from REE's, niobium and tantalum were identified in slag formed during tin smelting in numerous small and primitive metallurgical plants located on the Indonesian Tin Islands. The price of these elements is so attractive that future slag reprocessing seems to be profitable.

4. The Indonesian legal system currently forbids selling bulk tailings; therefore, cassiterite concentrate producers collect residual tailing at mine waste dumps. Only monomineral concentrate of a purity above $99.9 \%$, or the final product, i.e. extracted elements or their mixture, can be sold outside mining areas. Thus the mine operators would have to build processing plants within the mines in order to legally sell the product.

5. The dispersion and small scale of active open pit mines and illegal mining cause difficulties in estimating the potential REE resources in cassiterite deposits. Nevertheless, one can estimate that annually a minimum of 10,000 tons of minerals containing REE's (both LREE's-monazite and HREE's-xenotime) can be further processed.

6. Indonesian authorities are open to cooperating with foreign mining companies. This means that it is possible to open and mine new sources for REE minerals, and to gradually reduce the current scale of the Chinese monopoly. This is particularly important considering the efforts to guarantee domestic and international mineral security undertaken by the European Union and the United States.

\section{REFERENCES}

Critical raw materials for the EU. 2010 - Report of the Ad-hoc Working Group on defining critical raw materials Raw Materials Supply Group, Brussels, June 2010.

Di11 H.G., 2007 - Grain morphology of heavy minerals from marine and continental placer deposits, with special reference to Fe-Ti oxides. Sedimentary Geology 198, 1-27. 
Dill et al. 2006 - Dill H.G., Melcher F., F üß1 M., Weber B., 2006 - Accessory minerals in cassiterite A tool for provenance and environmental analyses of colluvial-fluvial placer deposits (NE Bavaria, Germany). Sedimentary Geology 191, 171-189.

Dill et al. 2012 - Dill H.G., W e ber B., Klosa D., 2012 - Morphology and mineral chemistry of monazite-zircon-bearing stream sediments of continental placer deposits (SE Germany): Ore guide and provenance marker. Journal of Geochemical Exploration 112, 322-346.

EU Resolution 2011: An effective raw materials strategy for Europe. European Parliament resolution of 13 September 2011 http://eur lex.europa.eu/LexUriServ/LexUriServ.:2013:051E:0021:0037:EN:PDF

F örster H.J., 1998 - The chemical composition of REE-Y-Th-U-rich accessory minerals in peraluminous granites of the Erzgebirge-Fichtelgebirge region, Germany. Part II: Xenotime. American Mineralogist 83, 1302-1315.

Galos et al. 2012a - Galos K., Nieć M., Radwanek-Bąk B., S makowski T., Szamałek K., 2012aBezpieczeństwo surowcowe Polski - ocena sytuacji w zakresie kopalin nieenergetycznych. Biul. PIG 452, $33-42$.

Galos et al. 2012b - Galos K., Nieć M., R adwanek-Bąk B., S makowski T., Szamałek K., 2012bBezpieczeństwo surowcowe Polski - bariery pokrycia krajowych potrzeb surowcowych w zakresie kopalin nieenergetycznych. Biul. PIG 452, 53-58.

Galos et al. 2012c - Galos K., Nieć M., Radwanek-Bak B., S makowski T., Szamałek K., 2012cBezpieczeństwo surowcowe Polski w Unii Europejskiej i na świecie. Biul. PIG 452, 43-52.

Gawęda et al. 2009 - G aw ęd a A., Krzemińs k a E., Wis zniew sk a J., 2009-Granity typu A w kompleksie mazurskim - przyczynek do dyskusji o klasyfikacji granitów. Przegląd Geologiczny 57, 478-488.

Geological map of Bangka 1:250,000, Northern part S. Andi Mangga and B. Djamal (1994), Southern part U. Margono, RJB. Supandjono \& E. Partoyo (1995), Geological Research and Development Centre Draw by Cartography, Geological Survay of Indonesia.

Kato et al. 2011 - Kato Y., Fujinaga K., Nakamura K., Takaya Y., Kitamura K., Ohta J., Toda R., Nakashima T., Iwamori H., 2011 - Deep-sea mud in the Pacific Ocean as a potential resource for rare-earth elements. Nature Geoscience 4, 535-539.

Kositcin et al. 2003 - Kositcin N., McNaughton N.J., Griffin B.J., Fletcher I.R., Groves D.I., R a s mussen B., 2003 - Textural and geochemical discrimination between xenotime of different origin in the Archaean Witwatersrand Basin, South Africa. Geochimica et Cosmochimica Acta 67, 709.

Maruejol et al. 1990 - Maruejol P., Cuney M., Turpin L., 1990 - Magmatic and hydrothermal R.E.E. fractionation in the Xihuashan granites (SE China). Contrib Mineral Petrol 104, 668-680.

Mining com. 2013a - http://www.mining.com/us-digging-through-waste-to-uncover-rare-earths

Mining com. 2013b - http://www.mining.com/avalon-rare-metals-lead-mad-surge-in-rare-earth-juniors

Mogilevsky et al. 2006 - Mogilevsky P., Zaretsky E.B., Parthasarathy T.A., Meisenkothen F., 2006 - Composition, lattice parameters, and room temperature elastic constants of natural single crystal xenotime from Novo Horizonte. Phys Chem Minerals 33, 691-698

Morton C.A., Hallsworth CR., 1999 - Processes controlling the composition of heavy mineral assemblages in sandstones. Sedimentary Geology 124, 3-29.

Ni et al.1995 - Ni Y., Hughes J.M., Mariano A.N., 1995 - Crystal chemistry of the monazite and xenotime structures. American Mineralogist 80, 21-26.

R a d w a n e k-B ą k B., 2011 - Zasoby kopalin Polski w aspekcie oceny surowców krytycznych Unii Europejskiej. Mineral Resources Management (Gospodarka Surowcami Mineralnymi) t. 27, z. 1, 5-19.

Schwartz et al. 2012 - Schwartz M.O., Rajah S.S., Askury A.K., Putthapiban P., Djaswadi S., 1995 - The Southeast Asian tin belt. Earth-Science Reviews 38, 95-293.

Sevastjanova et al. 2012 - Sevastjanova I., Hall R., Alderton D., 2012 - A detrital heavy mineral viewpoint on sediment provenance and tropical weathering in SE Asia. Sedimentary Geology 280, 179-194.

S makowski T., 2011 - Surowce mineralne - krytyczne czy deficytowe dla gospodarki UE i Polski. Zeszyty Naukowe IGSMiE PAN nr 81, Kraków, 59-68.

S za małek K., 2011a - Bezpieczeństwo surowcowe państwa. W: Bilans perspektywicznych zasobów kopalin Polski. Praca zbiorowa pod red. S. Wołkowicz, T.S makowski, S. Speczik. PIG-PIB Warszawa, s. 7-11.

S z a m a łek K., 2011b - Rational mineral deposit management in the light of mineral resources theory. Mineral Resources Management (Gospodarka Surowcami Mineralnymi) t. 27, nr 4, s. 5-15. 
Szamałek et al. 2011 - Szamałek K., Marcinowska A., Nejbert K., Speczik S., 2011 - Sea-floor massive sulphides from the Galapagos Rift Zone - mineralogy, geochemistry and economic importance. Geol.Quarterly, 55 (3), 187-202.

Szamałek et al. 2013 - Szamałek K., Konopka G., Zglinicki K., 2013 - Minerały i pierwiastki towarzyszące rudom kasyterytowym w Indonezji. Mat. 3 Konf. Międz. Metale towarzyszące w przemyśle metali nieżelaznych. Wrocław 15-17.05.2013. Streszczenia referatów, s. 10. IMN Gliwice, KGHM Ecoren.

Van Emden et al. 1997 - Van Emden B., Thornber M.R., Graham J., Lincoln F.J., 1997 - The Incorporation of Actinides in Monazite and Xenotime from Placer Deposits in Western Australia. Canadian Mineralogist, 35, 95-104.

Zhu X.K., O'Nions R.K., 1999 - Monazite chemical composition: some implications for monazite geochronology. Contrib Mineral Petrol 137, 351-363.

http://minerals.usgs.gov/minerals/pubs/commodity/tin/mcs-2013-tin.pdf

\section{NOWE POTENCJALNE ŹRÓDLO PIERWIASTKÓW ZIEM RZADKICH}

Słowa kluczowe

REE, HREE, LREE, monacyt, ksenotym, metale krytyczne

\section{Streszczenie}

Pierwiastki ziem rzadkich (REE) są niezwykle istotnym, choć rzadko występującym zasobem mineralnym odgrywającym ważną rolę w gospodarkach krajów wysoko rozwiniętych. Zakłócenia na międzynarodowym rynku surowcowym metali ziem rzadkich związane $\mathrm{z}$ monopolem Chin $\mathrm{w}$ zakresie ich produkcji i podaży zmuszają państwa wysoko rozwinięte do podejmowania intensywnych działań dla znalezienia nowych źródeł tych pierwiastków (wśród nich odpadów poprzeróbczych). W artykule przedstawiono wyniki badań składu mineralnego odpadów powstających na wyspie Bangka w trakcie wzbogacania koncentratu kasyterytowego. Badane próbki odpadów zawierają monacyt w ilości do 21,23 \%w oraz do 17,55 \%w ksenotymu (próbka z Sungkap, Bangka), ponadto występują w nich cyrkon, ilmenit, anataz, rutyl, pseudorutyl i kasyteryt. Poza pierwiastkami REE zidentyfikowano niob i tantal w żużlu powstającym podczas wytopu cyny. Autorzy oceniają, że rocznie możliwe jest dostarczanie do dalszej przeróbki minimum 10 tys. ton minerałów zawierających pierwiastki ziem rzadkich (zarówno lekkich LREE obecnych w monacycie, jak i ciężkich HREE w ksenotymie).

\section{NEW POTENTIAL SOURCE OF RARE EARTH ELEMENTS}

$$
\text { Key words }
$$

REE, HREE, LREE, monazite, xenotime, critical metals

\section{Abstract}

REE metals are a vital yet scarce resource which play a particularly significant role in developed countries and their technologically advanced economies. Disturbances in the international mineral commodities market for REE's caused by the Chinese supply monopoly force industrialized countries to launch intensive programs to discover new sources of these elements (even considering post-processing tailings). This paper discusses the mineral composition of tailings obtained in cassiterite extraction on Bangka Island. The analyzed tailing samples contain up to $21.23 \% \mathrm{w}$ monazite, up to $17.55 \% \mathrm{w}$ xenotime (Sungkap, Bangka), as well as zircon, ilmenite, anatase, rutile, pseurorutile, and cassiterite. Aside from REE's, niobium and tantalum were identified in slag formed during tin smelting. The authors estimate that annually a minimum of 10,000 tons of minerals containing REE's (both LREE's-monazite and HREE's-xenotime) can be further processed. 